\title{
Jurnal
}

Ilmu Manajemen dan Bisnis

Terakreditasi Kemenristekdikti Nomor 34/E/KPT/ Desember 2018

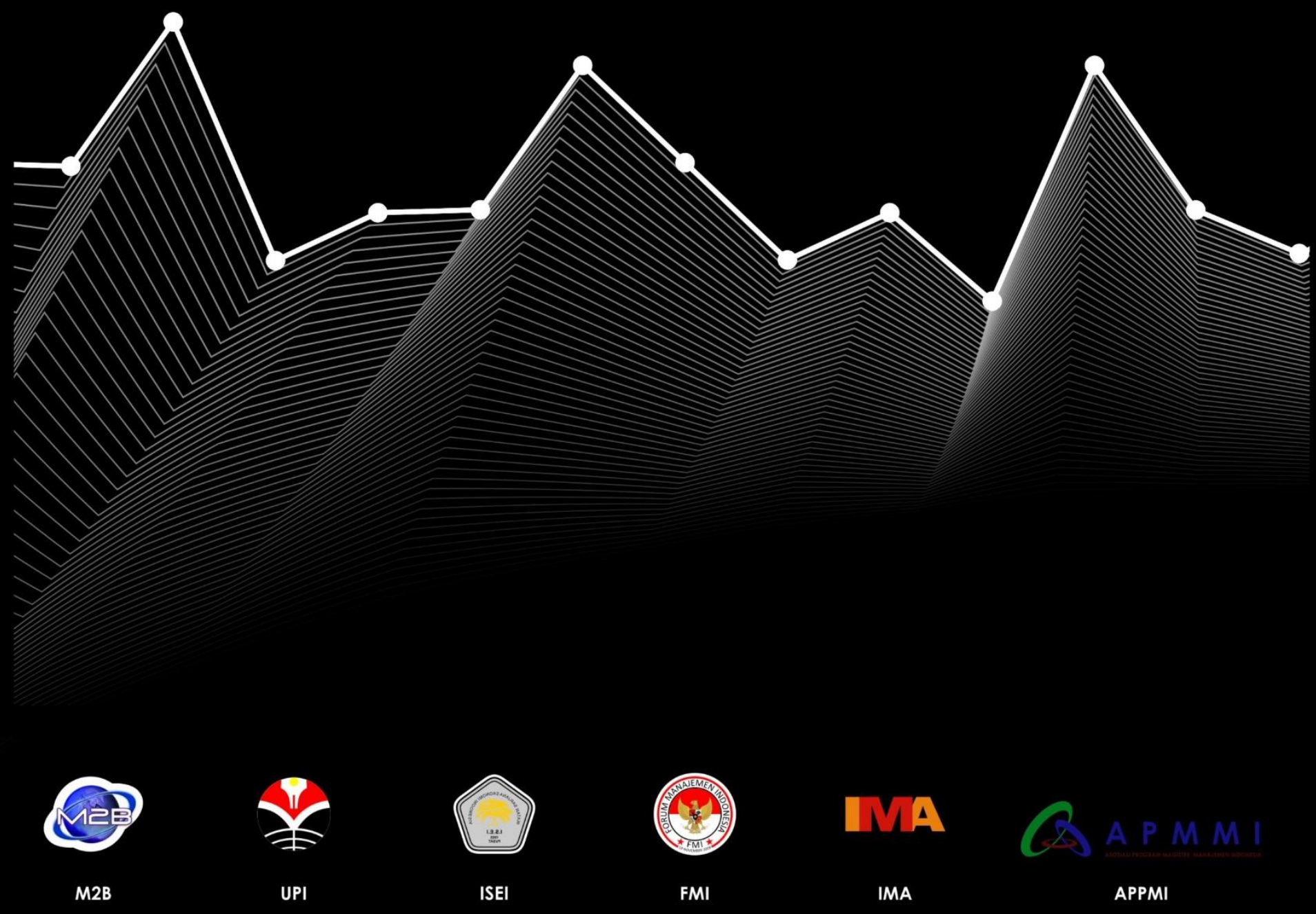




\section{AIM AND SCOPE}

The aim of this Jurnal Ilmu Manajemen dan Bisnis is to promote a principled approach to research on management science and business related concerns by encouraging inquiry into the relationship between theoretical and practical studies. Jurnal Ilmu Manajemen dan Bisnis, an electronic journal, provides a forum for publishing the original research articles, review articles from contributors, and the novel technology news related to management science and business.

Further, the journal is intended as an instrument for individuals conducting research on management science and business at both micro and macro levels of analysis as well as its relationship with other managerial and business functions.

Editorial Team welcome submissions of papers describing researchers, practitioners, regulators, students, and other parties interested in the development of management science and business. Accepts manuscripts of either quantitative research, qualitative research, mix method research, and Research and Development (R\&D) written in either Bahasa Indonesia or English.

The scopes of the topics include OrganzationL Behavior, Leadership, Human Resources Management, Innovation, IT, Operations and Supply Chain Management, Marketing Management, Financial Management, Accounting, Strategic Management, Entrepreneurship, and Green Business.

\section{PUBLICATION INFORMATION}

Jurnal Ilmu Manajemen dan Bisnis with registered number ISSN 2337-411X (Print) and ISSN 2503-3522 (Online), is a peer-reviewed journal published two times a year (March and September) by Management Program Sekolah Pascasarjana Universitas Pendidikan Indonesia. Jurnal Ilmu Manajemen dan Bisnis is intended to be the journal for publishing articles reporting the results of research on business. Jurnal Ilmu Manajemen dan Bisnis cooperation with Ikatan Sarjana Ekonomi Indonesia (ISEI) Jawa Barat, Forum Manajemen Indonesia (FMI), Indonesia Marketing Associations, Asosiasi Pengelola Magister Manajemen Indonesia (APMMI). DOI Prefix 10.17509/jimb by Crossref

\section{OPEN ACCES POLICY}

The article submitted to this online journal will be peer-reviewed at least 2 (two) reviewers. This journal provides immediate open access to its content on the principle that making research freely available to the public supports a greater global exchange of knowledge. Before going to review process, all manuscripts will be checked that they are free from plagiarism practice using "Turnitin" software. If there an indication of plagiarism, the manuscript will instantly be rejected. Indeks by Google Scholar and Indonesia One Search 


\section{EDITORIAL BOARD}

\section{Editor in Chief}

Prof. Dr. Hj. Ratih Hurriyati, MP, ID SCOPUS : 57074106300, Fakultas Pendidikan Ekonomi dan Bisnis, Universitas Pendidikan Indonesia, Indonesia, Indonesia

\section{Vice Editor}

Prof. Dr. H. Agus Rahayu, MP, ID SCOPUS : 57188870250, Fakultas Pendidikan Ekonomi dan Bisnis, Universitas Pendidikan Indonesia, Indonesia, Indonesia, Indonesia

Prof. Dr. H. Disman MS, ID SCOPUS : 57188870250, Fakultas Pendidikan Ekonomi dan Bisnis, Universitas Pendidikan Indonesia, Indonesia, Indonesia

\section{Editor}

Dr. Vanessa Gaffar, SE., Akt., MBA, ID SCOPUS : 57193739131, Fakultas Pendidikan Ekonomi dan Bisnis, Universitas Pendidikan Indonesia, Indonesia, Indonesia

Dr. Lili Adi Wibowo, S.Pd., S.Sos., MM, ID SCOPUS : 57193737268, Fakultas Pendidikan Ekonomi dan Bisnis, Universitas Pendidikan Indonesia, Indonesia, Indonesia, Indonesia

Dr. Heny Hendrayati, MM, ID SCOPUS : 57193444685, Fakultas Pendidikan Ekonomi dan Bisnis, Universitas Pendidikan Indonesia, Indonesia

Dr. Yana Setiawan, S.Pd., MM, Fakultas Pendidikan Ekonomi dan Bisnis, Universitas Pendidikan Indonesia, Indonesia, Indonesia

\section{Web and Layout Editor}

Sulastri S.Pd., M.Stat., MM, Pendidikan Kewirausahaan, Univesitas Pendidikan Indonesia, Indonesia

\section{Circulation and Distribution}

Usep Muharam, S.Pd, Program Studi Manajemen, Universitas Pendidikan Indonesia 


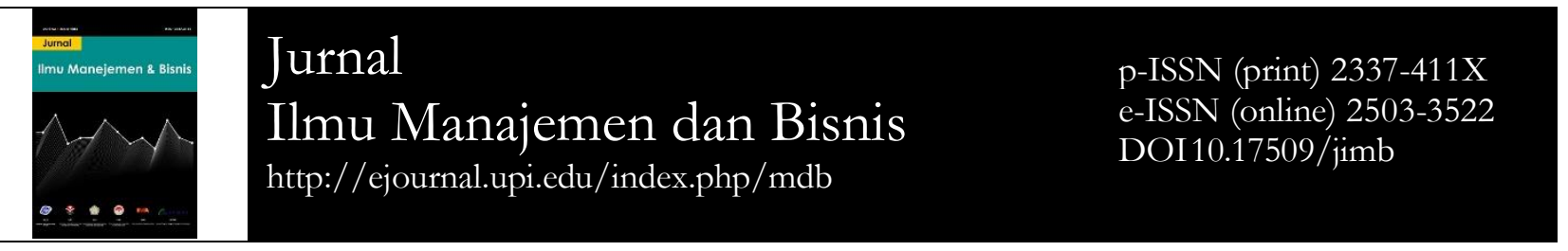

\section{PEER REVIEWER}

Prof. Ikuro Yamamoto, Kinzo University, Japan

Prof. Je Dae SIK, Youngsan University, South Korea

Prof. Kim, Tae Hee, Youngsan University, South Korea

Prof. Lincoln Arsyad, M.Sc., Ph.D, SCOPUS ID: 54683353100, Fakultas Ekonomi dan Bisnis, Universitas Gajah Mada, Indonesia

Prof. Dr. Sukrisno Agoes., Ak., MM., CPA, ID SCOPUS :54683353100, Fakultas Ekonomi, Universitas Tarumanagara, Indonesia

Ina Primiana, ID SCOPUS: 55437876800 Fakultas Ekonomi dan Bisnis, Universitas Padjadjaran, Indonesia

Dr. Ir. H. Arry Akhmad Arman, MT, ID SCOPUS : 56039352800 Sekolah Teknik Elektro dan Informatika, Institut Teknologi Bandung, Indonesia

Dwi Larso, Ph.D M.Sc, ID SCOPUS : 34969340900, School of Business and Management,Institut Teknologi Bandung, Indonesia

Ari Waroka, Ph.D., M.Sc, MDEM., MCEUE, DEA, ID SCOPUS : 55385462400, Universidad Autonoma de Madrid, Madrid, Spain 
The Effect Of Salesperson Ethical Behavior on Satisfaction, Trust and Customer Loyalty 121-130 Putu Bagus Wedatama ${ }^{1}$, I Putu Gde Sukaatmadja ${ }^{2}$

Analisis Penerapan Program Laku Pandai pada PT XYZ

Putu Ayu Sruti Permata Sari ${ }^{1}$, Budi Frensidy ${ }^{2}$

Evaluasi Penerapan PSAK 69 'Agrikultur’ atas Aset Biologis pada Perusahaan Sawit PT X 143-152 Adhi Nugraha ${ }^{1}$, Aruna Wirjolukito ${ }^{2}$

Analysis of Organization Culture With Denison's Model Approach For Automotive 153-166 Company in Indonesia

Pebri Tutur Srihadi ${ }^{1}$, Ferdinand Dehoutman Saragih ${ }^{2}$

Three Lines of Defense dalam Penerapan Manajemen Risiko pada Perusahaan Niaga 167-180 Gas Bumi

Iffah Nabilah Sihab ${ }^{1}$, Vera Diyanti ${ }^{2}$

Analisis Kelayakan Usaha Street Food Untuk Pemanfaatan Lahan Parkir Pasar Modern

Rangga Herbowo Putra ${ }^{1}$, Endang Chumaidiyah, Meldi Rendra ${ }^{2}$

Stabilitas Bank : Sebuah Pengujian Berdasarkan Teori Resource Based View

The Business Model Canvas as A Solution of Innovation For A Small Business

Formulasi Perancangan Strategi Pengembangan Usaha Menggunakan Analisis SWOT 205-212 dan Business Model Canvas

Fanji Wijaya

Strategi Pengembangan Model Bisnis Online Shop Zavair Scraves dengan menggunakan 213-222 pendekatan BCG Matrix

Herlina Sari

Dynamic Capabilities in The Creative Arts Industry

Ghia Ghaida Kanita ${ }^{1}$, Resa Respati ${ }^{2}$

Minat Berwirausaha Mahasiswa

Arief Budiman ${ }^{1}$, Heny Hendrayati ${ }^{2}$, Yoga Perdana ${ }^{3}$, Eka Surachman ${ }^{4}$ 